\title{
Hypophosphatemia in the Intensive Care Unit: Incidence, Predictors and Management.
}

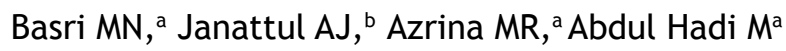 \\ aDepartment of Anaesthesiology and Intensive Care, Kulliyah of Medicine, International \\ Islamic University Malaysia, Kuantan, Pahang, Malaysia.

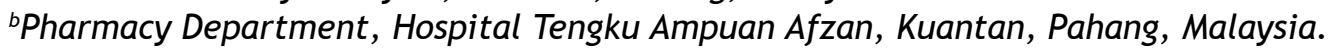

\begin{abstract}
Introduction: Our objectives are to identify the incidence of hypophosphatemia and the associated risk factors. We also want to establish intravenous replacement therapy that is effective for ICU patients. Methods: A prospective observational study assessing adults admitted to ICU in between March and May 2009. All patients without baseline phosphate level and renal failure were excluded. They were evaluated for the occurrence of common risk factors. Association with independent variables that includes age, gender and BMI were verified. Evaluation of IV replacement therapy was done in the treated patients. Results: From 50 patients that were reviewed, nine were excluded. There were $66 \%$ male and $34 \%$ female with mean age $46.88 \pm 17.89$. The mean ICU stay was $8.00 \pm 6.41$ days. The incidence of hypophosphatemia was $29 \%(n=12 / 41)$. Gender and creatinine clearance was found to be significantly different between normophosphatemia and hypophosphatemia patients. There was no significant association for each potential risk factor and the number of risk factors $(\geq 3)$ with the incidence of hypophosphatemia. Multi-linear regression analysis showed that lactate, creatinine clearance and $\mathrm{pH}$ were significant predictors to the serum levels. A significant difference of mean serum phosphate was seen after repletion by total dose of 10, 20 and 40 mmols in the treatment subgroups. Conclusions: The incidence of hypophosphatemia in our ICU was high and comparable to previous studies. None of the commonly reported risk factors is associated with hypophosphatemia in this studied population. Among all significant correlated variables, only $\mathrm{pH}$ was found to be a significant predictor for serum phosphate. Baseline phosphate level may guide the initial replacement dose to prevent delay in normalization of serum levels.
\end{abstract}

KEYWORDS: Hypophosphatemia, incidence, risk factors, management

\section{INTRODUCTION}

Hypophosphatemia is a metabolic disorder that is commonly encountered in critically ill patients. The incidence in critically ill patients had been reported ranging from $20 \%$ to $40 \%$, reaching up to $80 \%$ in trauma patients. ${ }^{1}$ Hypophosphatemia is defined as plasma phosphate level below $0.80 \mathrm{mmol}$ per litre ( $\mathrm{mmol} / \mathrm{L})$, and can be further divided into subgroups of mild (a plasma phosphate of 0.66 to $0.79 \mathrm{mmol} / \mathrm{L}$ ), moderate (plasma phosphate of 0.32 to 0.65 $\mathrm{mmol} / \mathrm{L}$ ) and severe (plasma phosphate of less than $0.32 \mathrm{mmol} / \mathrm{L}) .2,3$ Inadequate intake, redistribution of phosphate into cells and loss of phosphate from the body are the main mechanisms related to the

Corresponding author:

Mohd Basri Mat Nor

Department of Anaesthesiology and Intensive Care, Kulliyah of Medicine, International Islamic

University Malaysia,

Kuantan, Pahang, Malaysia

Phone: +6019 9822105

Fax: +609 5043402

Email address: basri.matnor@gmail.com occurrence. ${ }^{4}$ Phosphate has many roles in physiological functions, thus the depletion of serum phosphate could lead to impairment in multiple organ systems, which include respiratory system, cardiovascular system, neurological system, muscular system, hematological and metabolic functions. ${ }^{3,}{ }^{4}$ In critically ill patients, factors such as sepsis, trauma, fluid therapy, refeeding syndrome, acid-base disorders, medications and renal replacement could contribute to the occurrence of hypophosphatemia. ${ }^{1}$

The management of hypophosphatemia, especially in critically ill patients is crucial. Intravenous (IV) phosphate, given as sodium phosphate, potassium phosphate or glucose phosphate has been the management for phosphate repletion in Intensive Care Unit (ICU). 5-10 Generally, rapid administration of high-dose IV phosphate can lead to adverse effects such as hypotension, hypocalcaemia and renal failure. ${ }^{1,4}$ Therefore, it is always a concern with the amount and rate of administration of IV phosphate during phosphate repletion therapy. Multiple dosing strategies had been evaluated in order to achieve rapid normalization of serum phosphate level, with avoidance of its associated adverse effects..$^{5-10}$ All these studies had shown a successful repletion with 
their dosage regimen without any reported serious adverse event. However, the studies were carried out from different ICU settings that limit the generalization of the recommended dosing protocol to other populations.

Incidence of hypophosphatemia in critically ill patients in Malaysia has not been reported before. We aimed to report the incidence of hypophosphatemia, and to identify possible risk factors for hypophosphatemia in critically ill patients in Malaysia. We also aimed to establish a more rational IV phosphate repletion regimen specific to this population.

\section{MATERIALS AND METHOD}

This prospective observational study was approved by the local Research Ethics Committee, and registered with the National Medical Research Register of Ministry of Health, Malaysia (NMRR ID: 09-208-3782). This study was conducted in the Intensive Care Unit (ICU) of Hospital Tengku Ampuan Afzan, Kuantan, a tertiary hospital located in east cost of Malaysia between $1^{\text {st }}$ of April 2009 to $31^{\text {st }}$ of May 2009. All adult patients (aged $>18$ years old) admitted to ICU within the study period were considered for entry into the study. Patients without the phosphate baseline level, diagnosed as renal failure, chronic renal failure with or without dialysis were excluded from the study.

Plasma phosphate levels were measured by Olympus AU2700 analyzer (Olympus America Inc.). Normal plasma phosphate levels range between 0.80 $\mathrm{mmol} / \mathrm{L}$ and $1.45 \mathrm{mmol} / \mathrm{L}$. Hypophosphatemia is definedbyplasmaphosphatelevellessthan $0.80 \mathrm{mmol} / \mathrm{L}$, and it can further be divided into mild, moderate and severe based on previous classification. ${ }^{2,3}$

Patients were categorized into two groups, based on their baseline serum phosphate level, normophosphatemia and hypophosphatemia. Hypophosphatemic patients were further categorized into three differentsubgroups mild, moderate and severe hypophosphatemia. ${ }^{2,3}$ Demographic profiles, including age, gender, race, height, weight, admission diagnosis, past medical history and concurrent medications were extracted from clinical records.

Routine laboratory monitoring included renal profile, liver function test, full blood count, arterial blood gases, coagulation profile, calcium, magnesium and phosphate were also extracted from clinical records. Nutritional support, prior to and during admission, including type of nutrition support formulation, were also recorded.

The presence of commonly reported risk factors of hypophosphatemia such as sepsis, diuretic therapy, nutritional support, alcohol withdrawal, blood transfusion, insulin therapy, diabetes, intravenous glucose administration and steroid therapy ${ }^{1,4}$ were also evaluated. Sepsis was defined by the ICU physician and required two or more systemic inflammatory response syndrome (SIRS) criteria, and suspected or confirmed bacterial or viral infection.

In patients who received IV phosphate repletion therapy, the initial serum phosphate level before (pre-level) and after (post-level) administrations of phosphate replacement were recorded. The dose of IV phosphate and sampling time were also recorded.

\section{Statistical Analysis}

Statistical analysis was performed using SPSS version 15.0 (IBM, Somers, New York, USA) and Microsoft Excel 2007 (Microsoft Incorporation, Redmond, Washington, USA). Results were presented as mean \pm standard deviation. Comparisons of variables between the two groups were analyzed using the independent or paired t-test for normally distributed variables or the Mann-Whitney test for non-parametric variables.

Categorical variables were compared with chi square or Fisher's exact test. Association of creatinine clearance, arterial $\mathrm{pH}$ and lactate levels with the serum phosphate level were analyzed using Spearman's correlation. Risk factors for hypophosphatemia were further calculated using the multivariable logistic regression model. Statistically significant was considered when the $p$ value was $<0.05$.

\section{RESULTS}

Fifty patients were screened during the study period for inclusion into the study. Nine patients were excluded as they were diagnosed with renal failure and with markedly high serum phosphate level. Five patients were diagnosed with acute renal failure, while 3 patients were diagnosed with chronic renal failure and one patient with end stage renal failure. Mean baseline serum phosphate level for these patients were $2.5 \pm 0.9 \mathrm{mmol} / \mathrm{L}$. Therefore, they were excluded from the study.

From the 41 patients analyzed, 12 patients (29\%) hypophosphatemia, of which 4 (33\%) of them with mild hypophosphatemia, 5 (42\%) with moderate hypophosphatemia and 3 (25\%) with severe hypophosphatemia. The demographic characteristics of patients with hypophosphatemia and normophosphatemia were compared and presented in Table I.

There was no difference in the mean age of the hypophosphatemic and normophosphatemic patients, $(40 \pm 13$ versus $50 \pm 18 ; \quad p=0.10)$. However, hypophosphatemia was more common in female patients $(67 \%)$ compared to male patients $(33 \%)$, $(p=0.01)$. There were no differences found in distribution of patients according to race between hypophosphatemic and normophosphatemic patients $(p=0.07)$. 
Table I: Demographic data

\begin{tabular}{llcll}
\hline Variables & $\begin{array}{l}\text { Cohort } \\
(\mathrm{n}=41)\end{array}$ & $\begin{array}{l}\text { Normophosphatemia } \\
(\mathrm{n}=29)\end{array}$ & $\begin{array}{l}\text { Hypophosphatemia } \\
(\mathrm{n}=12)\end{array}$ & $\mathrm{P}$ \\
\hline $\begin{array}{l}\text { Age (years) } \\
\text { Gender }\end{array}$ & $47 \pm 18$ & $50 \pm 18$ & $40 \pm 13$ & 0.10 \\
Male & $27(66 \%)$ & $23(79 \%)$ & $4(33 \%)$ & 0.01 \\
$\begin{array}{l}\text { Female } \\
\text { Race }\end{array}$ & $14(34 \%)$ & $6(21 \%)$ & $8(67 \%)$ & \\
Malay & & & & \\
Chinese & $33(81 \%)$ & $24(82 \%)$ & $9(75 \%)$ & 0.07 \\
Indian & $5(12 \%)$ & $3(10 \%)$ & $2(17 \%)$ & \\
Others & $1(2 \%)$ & $1(4 \%)$ & $0(0 \%)$ & 0.48 \\
ICU stay (days) & $2(5 \%)$ & $1(4 \%)$ & $1(8 \%)$ & 0.92 \\
Body mass index & $8.0 \pm 6.4$ & $8.9 \pm 7.3$ & $7.9 \pm 5.0$ & 0.02 \\
(BMI) (kg/m2) & $25.5 \pm 6.5$ & $25.6 \pm 6.6$ & $25.4 \pm 6.6$ & 0.44 \\
Creatinine clearance & & & & \\
(ml/min) & $64.4 \pm 37.5$ & $55.3 \pm 32.2$ & $84.3 \pm 41.6$ & 0.50 \\
Albumin (g/L) & $28.3 \pm 6.9$ & $28.8 \pm 7.8$ & $26.9 \pm 4.0$ & \\
Protein (g/L) & $58.8 \pm 10.3$ & $59.5 \pm 10.9$ & $57.1 \pm 8.8$ & \\
\hline Data expressed as mean & & &
\end{tabular}

Data expressed as mean $\pm \mathrm{SD}$, or $\mathrm{n}(\%)$

\section{Risk factors for hypophosphatemia}

All patients had sepsis, 12 patients received diuretic therapy, 17 patients received nutritional support (enteral feeding), one patient with alcohol withdrawal, five patients received blood transfusion, 15 patients received insulin therapy, 12 patients with underlying diabetes, 17 patient received glucose administration and 7 patients received steroid therapy. The risk factors presented in patients with hypophosphatemia and normophosphatemia were compared and presented in Figure 1. There were no differences in the percentage of patients with the above risk factors between those with hypophosphatemia and normophosphatemia.

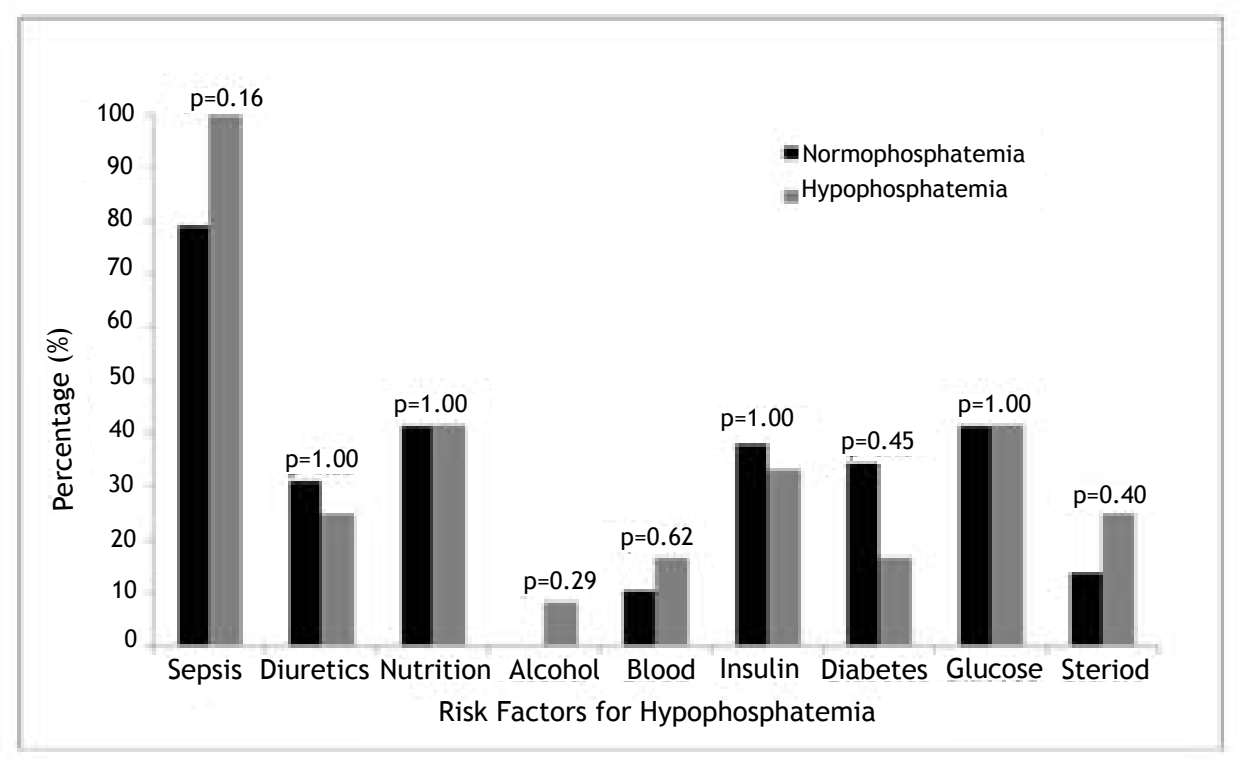

Figure 1: Commonly reported risk factors for hypophosphatemia in normophosphatemia and hypophosphatemia patients were analyzed using Fisher-exact test 
Twenty four patients $(59 \%)$ presented with less than three risk factors, and 17 patients (41\%) presented with more than three risk factors. There was no association found between the number of risk factors with the incidence of hypophosphatermia (less than three risk factors versus more than three risk factors, $p=0.73$ ). The correlation between creatinine clearance,arterial $\mathrm{pH}$ and lactate levels with the serum phosphate level were also evaluated. Serum lactate levels, creatinine clearance and arterial pH correlated with the serum phosphate level (Table II). However, multivariable logistic regression analysis showed that only $\mathrm{pH}$ predicted the serum phosphate level, $F(6,29)=4.612, R 2$ is $>0.14, p=0.04$.

Table II: Correlation of creatinine clearance, arterial pH, and lactate level with serum phosphate level

\begin{tabular}{lcl}
\hline Variables & r value & p value \\
\hline Creatinine clearance & -0.39 & 0.01 \\
pH & -0.43 & 0.01 \\
Lactate level & 0.30 & 0.03 \\
\hline
\end{tabular}

Correlation coefficients $(r)$ were calculated using Spearman correlation

\section{Intravenous (IV) phosphate replacement therapy}

Six patients who received IV phosphate injection were analyzed for the efficacy of the therapy. $10 \mathrm{mmol}$ of potassium phosphate diluted in $50 \mathrm{ml} 0.9 \%$ sodium chloride (normal saline) were administered within one hour. Normalization in serum phosphate levels were seen after repletion with a total dose of 10,30 and $40 \mathrm{mmol}$ of IV phosphate, $(\mathrm{p}=0.002)$. Four patients needed only one dose of $10 \mathrm{mmol}$ of IV phosphate, whereas two patients needed repeated IV phosphate repletion therapy (Patient 4 and Patient 6). The pre-and post-infusion serum phosphate levels are presented in Table III.

Table III: Pre- and post-infusion serum phosphate level after phosphate therapy

\begin{tabular}{lcll}
\hline Patient & $\begin{array}{l}\text { Phosphate repletion } \\
\text { therapy }\end{array}$ & $\begin{array}{l}\text { Preinfusion } \\
(\mathrm{mmol} / \mathrm{L})\end{array}$ & $\begin{array}{l}\text { Postinfusion } \\
(\mathrm{mmol} / \mathrm{L})\end{array}$ \\
\hline 1 & $10 \mathrm{mmol}$ & 0.73 & 1.12 \\
2 & $10 \mathrm{mmol}$ & 0.27 & 0.48 \\
3 & $10 \mathrm{mmol}$ & 1.05 & 1.13 \\
4 & $10 \mathrm{mmol}$ & 0.50 & 0.64 \\
& $+10 \mathrm{mmol}$ & 0.64 & 0.88 \\
5 & $+10 \mathrm{mmol}$ & 0.45 & 1.02 \\
6 & $10 \mathrm{mmol}$ & 0.41 & 0.68 \\
& $10 \mathrm{mmol}$ & 0.31 & 0.34 \\
& $+10 \mathrm{mmol}$ & 0.34 & 0.36 \\
& $+10 \mathrm{mmol}$ & 0.34 & 0.60 \\
\hline
\end{tabular}

Corrected serum calcium concentrations were maintained in all patients between 2.13 and 2.43 $\mathrm{mmol} / \mathrm{L}$ during the phosphate repletion therapy. Neither serum magnesium nor serum potassium concentrations changed significantly above or below the normal range. No patient had a peak serum phosphate level $>1.45 \mathrm{mmol} / \mathrm{L}$. No other adverse effects were demonstrated in these patients.

\section{DISCUSSION}

To our knowledge, this is the first study to describe the incidence of hypophosphatemia in Malaysian population. Overall, the incidence of hypophosphatemia in the studied population was $29 \%$, similar to the previously reported incidence of hypophosphatemia in other ICU subjects (28 to $40 \%$ ). ${ }^{5,11}$ About $75 \%$ of the patients in this study had mild to moderate hypophosphatemia, while $25 \%$ had severe hypophosphatemia. Incidence of hypophosphatemia is varied in critically ill patients. ${ }^{1}$ It was reported that among hospitalized patients with hypophosphatemia, $45 \%$ of the incidence occurs in the ICU population. ${ }^{12}$ In critically ill patients, the incidence is expected to be higher due to the presence of multiple causal factors. However, the occurrence of hypophosphatemia in critically ill patients is much higher in certain patient groups especially in trauma patients, in which it has been reported to achieve as high as $100 \% .^{13}$

We reported no differences in age, racial distribution, number of ICU stay and baseline body mass index (BMI) between hypophosphatemic patients and normophosphatemic patients. In addition, there were also no 
differences in mean age, albumin levels and protein levels. These data suggested that the incidence of hypophosphatemia in our studied population probably is not associated with poor nutritional status. In patients with poor nutritional status, hypophosphatemia was commonly seen in patients with weight loss and low albumin levels. ${ }^{14} \mathrm{We}$ also showed that hypophosphatemia occurred more frequently in women $(67 \%)$ than in men $(33 \%)$. Hormonal change was shown to influence the serum phosphate level in women. ${ }^{15}$ Therefore, different in serum phosphate level between men and women are possibly seen. Hence, this could explain why hypophosphatemia is more common in women in this study.

In this study, all patients were evaluated for the presence of commonly reported risk factors as described in previous studies, which includes sepsis, use of diuretics, nutritional support, alcohol withdrawal, blood transfusion, insulin therapy, diabetes, intravenous glucose administration and steroid therapy. The number of risk factors presented was also studied (more or equal to 3 ) and was found to be not associated with the incidence of hypophosphatemia. Even though our finding did not demonstrate an association between commonly reported risk factors with the incidence of hypophosphatemia, we acknowledge that a proper study needs to be carried out to further evaluate these factors.

The association between hypophosphatemia with sepsis had been described previously. ${ }^{16}$ It was shown that serum phosphate level became normal within days after symptoms of acute sepsis subsided. Diuretic and steroid are among drugs that are commonly being used in ICU, that can cause urinary phosphate loss, ${ }^{3,4}$ thus can be one of the causes for hypophosphatemia. Hypophosphatemia related to refeeding syndrome may be seen in critically ill patients who have been starved or with poor nutritional status. ${ }^{11}$ It may occur be due to redistribution of phosphate into cells during initiation of nutritional support, either by enteral or parenteral route. The same mechanism also implied in patients who received intravenous glucose administration. ${ }^{3}$ Few studies have described the association between hypophosphatemia with alcohol withdrawal. ${ }^{17-19}$ Hypophosphatemia in alcoholic patients might be related to poor nutritional status (refeeding syndrome), diarrhea, increased urinary phosphate loss and direct toxic effect of alcohol on the proximal tubule. ${ }^{3}$ In general, decreased intestinal phosphate absorption, internal phosphate redistribution and increased urinary phosphate loss, are the main mechanisms that could lead to hypophosphatemia.

Correlation of serum phosphate with creatinine clearance, lymphocyte counts, arterial pH levels, serum lactate levels, magnesium and calcium levels were evaluated. Of these factors, only serum lactate levels, creatinine clearance and $\mathrm{pH}$ correlated with serum phosphate levels. In a multivariable regression analysis, $\mathrm{pH}$ was significantly predictive of serum Volume 11 Number 1 June 2012 phosphate levels. A correlation of $\mathrm{pH}$ with serum phosphate levels is related to the physiologic changes during alkalosis that may lower serum phosphate levels via transcellular shift. ${ }^{20,21}$ In respiratory alkalosis, decrease in carbon dioxide $\left(\mathrm{CO}_{2}\right)$ resulted in diffusion of intracellular $\mathrm{CO}_{2}$ across the cell membrane, into plasma. This resulted in a rise in $\mathrm{pH}$ that further stimulates glycolysis, in which phosphate ion is required to produce energy. Thus, circulating organic phosphate will shift across the cell membrane leading to a decrease in serum phosphate concentration. $^{21}$ This finding highlighted the importance of identifying a patient at risk factor for developing hypophosphatemia, so that a necessary preventive mechanism could be employed.

Hypophosphatemia can cause multiple organ dysfunctions, including respiratory, cardiovascular, hematologic, neurological, and muscular, which may result in mortality. Thus, phosphate repletion via IV phosphate therapy is very crucial, especially in critically ill patients. Phosphate may precipitate with calcium, therefore, rapid administration of high dose of IV phosphate may results in adverse event such as hypotension, hypocalcemia and renal failure. ${ }^{4}$ Many studies have evaluated a different dosage regimen of IV phosphate repletion in ICU. ${ }^{5-10}$ Early studies have suggested a small amount of phosphate, a total dose of $15 \mathrm{mmol}$ per hour $(\mathrm{mmol} / \mathrm{H})$ given in a longer rate of infusion up to 12 hours. ${ }^{6,7,22-24}$ Recent studies employed more aggressive regimes for phosphate repletion in ICU (up to $45 \mathrm{mmol}$ over three hours)..$^{8,10}$ These rapid and high-dose regimes have been used without any adverse effects, hence establishing several protocols. However, there are differences in the studies such as sample size, heterogenous population, general care and different preparation of IV phosphate (sodium phosphate and potassium phosphate), necessitates a local study in our setting, in order to evaluate our local practice in the phosphate repletion regimen.

In this study, six patients with baseline serum phosphate ranging from $0.31-1.05 \mathrm{mmol} / \mathrm{L}$, received IV phosphate $(10 \mathrm{mmol}$ for each repletion). Overall, there was an improvement of serum phosphate levels after the administration of IV phosphate and none of the patients encountered with any adverse event during the administration. Serum phosphate levels were normalized after a cumulative dose of 10,30 and 40 mmol of intravenous phosphate. Based on this finding, we proposed that the dose of intravenous phosphate could be based on the baseline serum phosphate. Higher dose $(40 \mathrm{mmol})$ can be given in patients with severe hypophosphatemia (serum phosphate level less than $0.32 \mathrm{mmol} / \mathrm{L}$ ), $10-30 \mathrm{mmol}$ can be given in patients with mild to moderate hypophosphatemia $(0.32-0.79 \mathrm{mmol} / \mathrm{L})$. This recommendation could assist clinicians in managing hypophosphatemia in our institution. There were other studies described similar dosing recommendation..$^{9,1}$

Our study does have several limitations. First, 
the sample size of this study was small, and it was conducted in a single center. Secondly, this was observational study, hence treatment, medication and general care were not standardized thus reduces the probability to generalize the data. Despite these limitations, we believe these preliminary findings from this study could assist in further studies in our local population.

\section{CONCLUSION}

Hypophosphatemia is an important and common metabolic disorder in critically ill patients. We demonstrated that hypophosphatemia occurred commonly in our local population, similar to other countries. There is a potential benefit in identifying patients at risk of hypophosphatemia, hence phosphate therapy could be immediately administered to prevent further complications. Further study is needed to suggest a proper dosing schedule in order to standardize method of practice in our institution.

\section{REFERENCES}

1. Geerse DA, Bindels AJ, Kuiper MA, et al. Treatment of hypophosphatemia in the intensive care unit: a review. Crit Care 2010;14:R147.

2. Knochel JP. The pathophysiology and clinical characteristics of severe hypophosphatemia. Arch Intern Med 1977;137:203-20.

3. Gaasbeek A, Meinders AE. Hypophosphatemia: an update on its etiology and treatment. Am J Med 2005;118:1094-101.

4. Bugg NC, Jones JA. Hypophosphataemia. Pathophysiology, effects and management on the intensive care unit. Anaesthesia 1998; 53:895-902.

5. Zazzo JF, Troché G, Ruel P, Maintenant $\mathrm{J}$. High incidence of hypophosphatemia in surgical intensive care patients: efficacy of phosphorus therapy on myocardial function. Intensive Care Med 1995;21:826-31.

6. Clark CL, Sacks GS, Dickerson RN, Kudsk KA, Brown RO. Treatment of hypophosphatemia in patients receiving specialized nutrition support using a graduated dosing scheme: results from a prospective clinical trial. Crit Care Med 1995;23:1504-11.

7. Rosen GH, Boullata JI, O'Rangers EA, Enow NB, Shin B. Intravenous phosphate repletion regimen for critically ill patients with moderate hypophosphatemia. Crit Care Med 1995; 23:1204-10.

8. Charron T, Bernard F, Skrobik Y, et al. Intravenous phosphate in the intensive care unit: more aggressive repletion regimens for moderate and severe hypophosphatemia. Intensive Care Med 2003;29:1273-8.

9. Taylor BE, Huey WY, Buchman TG, Boyle WA, Coopersmith CM. Treatment of hypophosphatemia using a protocol based on patient weight and serum phosphorus level in a surgical intensive care unit. J Am Coll Surg 2004;198:198-204.

10. French C, Bellomo R. A rapid intravenous phosphate replacement protocol for critically ill patients. Crit Care Resusc 2004;6:175-9.

11. Marik PE, Bedigian MK. Refeeding hypophos phatemia in critically ill patients in an intensive care unit. A prospective study. Arch Surg 1996;131:1043-7.

12. Hoffmann M, Zemlin AE, Meyer WP, Erasmus RT. Hypophosphataemia at a large academic hospital in South Africa. J Clin Pathol 2008;61:1104-7.

13. Berger MM, Rothen C, Cavadini C, Chiolero L. Exudative mineral losses after serious burns: a clue to the alterations of magnesium and phosphate metabolism. Am J Clin Nutr 1997;65:1473-81.

14. Kagansky N, Levy S, Koren-Morag N, Berger D, Knobler H. Hypophosphataemia in old patients is associated with the refeeding syndrome and reduced survival.J Intern Med 2005;257:461-8.

15. Cirillo M, Ciacci C, De Santo NG. Age, renal tubular phosphate reabsorption, and serum phosphate levels in adults. $N$ Engl $J$ Med 2008;359:864-6.

16. Riedler GF, Scheitlin W a. Hypophosphatae mia in septicaemia: higher incidence in gram-negative than in gram-positive infections. Br Med J 1969;1:753-6.

17. Larsson L, Rebel K, Sörbo B. Severe hypophosphatemia--a hospital survey. Acta Med Scand 1983;214:221-3.

18. King AL, Sica DA, Miller G, Pierpaoli S. Severe hypophosphatemia in a general hospital population. South Med J 1987;80:831-5.

19. Chermesh I, Meshulam C, Tamir A, Eliakim R, Levy Y. Hypophosphatemia in the hospitalized patient. Harefuah 2006;145: 786-8, 864.

20. Fisher J, Magid N, Kallman C, et al. Respiratory illness and hypophosphatemia. Chest 1983; 83:504-8.

21. O'Brien TM, Coberly L. Severe Hypophosphatemia in Respiratory Alkalosis. Adv Stud Med 2003;3:345-8.

22. Lentz RD, Brown DM, Kjellstrand CM. Treatment of severe hypophosphatemia. Ann Intern Med 1985;2:734.

23. Vannatta JB, Whang R, Papper S. Efficacy of intravenous phosphorus therapy in the severely hypophosphatemic patient. Arch Intern Med 1981;141:885-7.

24. Vannatta JB, Andress DL, Whang R, Papper $\mathrm{S}$. High-dose intravenous phosphorus therapy for severe complicated hypophosphatemia. South Med J 1983;76:1424-6. 